\title{
A model for hydrolytic degradation and erosion of biodegradable polymers
}

\author{
Kevser Sevim*, Jingzhe Pan \\ Department of Engineering, University of Leicester, Leicester LE1 7RH, UK \\ ks377@le.ac.uk,jp165@le.ac.uk
}

\begin{abstract}
For aliphatic polyesters such as PLAs and PGAs, there is a strong interplay between the hydrolytic degradation and erosion - degradation leads to a critically low molecular weight at which erosion starts. This paper considers the underlying physical and chemical processes of hydrolytic degradation and erosion. Several kinetic mechanisms are incorporated into a mathematical model in an attempt to explain different behaviours of mass loss observed in experiments. In the combined model, autocatalytic hydrolysis, oligomer production and their diffusion are considered together with surface and interior erosion using a set of differential equations and Monte Carlo technique. Oligomer and drug diffusion are modeled using Fick's law with the diffusion coefficients dependent on porosity. The porosity is due to the formation of cavities which are a result of polymer erosion. The model can follow mass loss and drug release up to $100 \%$, which cannot be explained using a simple reaction-diffusion. The model is applied to two case studies from the literature to demonstrate its validity. The case studies show that a critical molecular weight for the onset of polymer erosion and an incubation period for the polymer dissolution are two critical factors that need to be considered when predicting mass loss and drug release.
\end{abstract}

Keywords: Hydrolytic degradation; Erosion; Monte Carlo; Mathematical modelling; Drug release

\section{Statement of Significance}

In order to design bioresorbable implants, it is important to have a mathematical model to predict polymer degradation and corresponding drug release. However, very different behaviours of polymer degradation have been observed and there is no single model that can capture all these behaviours. For the first time, the model presented in this paper is capable of capture all these observed behaviours by switching on and off different underlying mechanisms. Unlike the existing reaction-diffusion models, the 
model presented here can follow the degradation and drug release all the way to the full disappearance of an implant.

\section{Introduction}

Biodegradable polymers, mainly aliphatic polyesters have highly desirable applications in the biomedical field, and presently being used as disposable products (e.g. syringe, blood bag), supporting materials (e.g. sutures, bone plates and sealant), artificial tissue/organs (e.g. artificial heart, kidney, eyes and teeth) and controlled release formulations for use with various drugs or hormones [1-4]. All these applications are primarily relied on the fact that the polymer ultimately disappears after providing a desired function [5,6]. In this respect, modelling their degradation and erosion in aqueous medium has attracted a great deal of attention in the literature [7]. According to the general knowledge in the literature, the degradation of the aliphatic polyesters is a chemical process which is resulted from polymer chain cleavage. The degradation products, monomers and oligomers, are released by diffusion. Grizzi, Garreau, Li and Vert [8] observed that thick PLA plates degrade faster than thin ones. They attributed this to acid accumulation which is more severe in a thick plate than that in a thin one. The carboxylic ends of the broken chains have a high degree of acid disassociation, which accelerates the polymer degradation. In thin sheets, this effect is significantly reduced because the oligomers can diffuse out quickly. Various mathematical models have been developed for polymer hydrolysis and erosion. Zygourakis et al. [9], Siepmann et al. [10] and Chen et al. [11] developed stochastic hydrolysis and erosion models to describe mass loss and drug release. However, these models did not consider the autocatalytic effect of the hydrolysis reaction, which has been recognised as the key factor for the degradation of PLAs and PGAs [12]. Recently Zhang et al. [13] developed a combined degradation and erosion model. This is an advanced model which follows oligomer diffusion at the continuum scale and random chain scissions and polymer erosion at the microscopic scale. However, the model is over-complicated for applications in practical device design. The important autocatalytic effect of the hydrolysis reaction was not considered, which means the model is unable to capture size effect of the degradation. Gopferich [14] suggested a two-phase mass loss profile for an eroding polymer. During the first phase, no 
significant mass loss can be obtained, while molecular weight is significantly reduced. This phase was designated as degradation. As soon as the molecular weight reaches a critical level at which the polymer becomes water soluble, an erosion phase initiates and the eroded material could be released into the surrounding medium leading to a large mass loss [15]. In their work a mechanism based link between the degradation and erosion processes was not made. Considering all the diffusion reaction models for polymer degradation, the mass loss predicted by the oligomer diffusion equation is generally less than $10 \%[16,17]$. However, the observed mass loss in the experimental literature is much higher even in the initial phase of degradation and can reach $100 \%$ at the end of degradation.

The objective of this paper is to combine a hydrolytic degradation model with a polymer erosion model so that the different behaviours of mass loss can be captured in a single model. The reaction-diffusion equations previously developed by Pan and his coworkers $[18,19]$ are adopted which consider the autocatalytic effect and are valid for aliphatic polyesters such as PLAs, PGAs and their copolymers. These equations are coupled mechanistically with an erosion model to provide a full picture of mass loss and drug release. It is shown that all the observed trends such as biphasic and triphasic mass losses can be obtained by using the combined model. Two case studies are provided to demonstrate that the model can fit with the experimental data satisfactorily. This paper focuses on amorphous polymers, which are the case for the experimental work cited in both case studies. Chapter four of reference [18] provides a model for polymer degradation considering chain-cleavage induced crystallisation.

\section{The mathematical model}

In this paper matter transport is limited to 2-dimensions although the mathematical model is generally valid for 3-dimensional problems. The case studies presented later in this paper can all be modelled under the assumption of 2-dimensional matter transport. We limit our effort to these cases for the sake of simplicity. A polymer implant is represented by using a set of regular pixels as shown in Fig. 1. The centres of the pixels correspond to nodes in a finite difference grid. Again, the model is not limited to this particular approach of discretization. In general, the finite element method can be used to solve the governing equations. However, for the sake of simplicity, we focus 
our effort on using the simplest discretization method. This allows us to demonstrate the capacity of the model before its extension to general polymer implants. The polymer morphology and pores are treated explicitly by using pixels of different state. As shown in Fig. 1, each pixel is identified by its indices $(i, j)$ in the $\mathrm{x}$ - and $\mathrm{y}$-directions. The state of each pixel is defined by its number averaged molecular weight, $M_{n}(i, j, t)$, erosion index, $S(i, j, t)$ and the local drug concentration $C_{d r u g}(i, j, t)$. The erosion index is defined as

$$
S(i, j, t)= \begin{cases}1 & \text { non-eroded pixel } \\ 0 & \text { eroded pixel }\end{cases}
$$

Water diffusion is a fast process in comparison to polymer degradation and erosion $[19,20]$. It is therefore assumed that water molecules are always abundant in the polymer. The hydrolytic degradation is reflected by the reduction in $M_{n}$ and polymer erosion is reflected by changes of $S(i, j, t)$ from 1 to 0 . The initial molecular weights of the pixels are set such that they follow a normal distribution using a direct Monte Carlo technique. A random number generator, $R$, is linked with a specified standard deviation to ensure that the molecular weight follows a normal distribution such that

$$
M_{n, 0}=M_{n, \text { mean }}+R \times M_{n, \text { st_dev }}
$$

in which $M_{n, \text { mean }}$ and $M_{n, s t}$ dev represent the mean value and standard deviation of the molecular weight distribution respectively.

\subsection{Model for hydrolytic degradation}

For hydrolytic degradation, the model developed by Pan and co-workers [18, 19] is used. A brief summary of their model is provided for the convenience of readers. The rate of polymer chain scission follows the governing equation $[18,19]$

$$
\frac{d R_{s}}{d t}=C_{e, 0}\left[1-\alpha\left(\frac{R_{s}}{C_{e, 0}}\right)^{\beta}\right]\left(k_{1}+k_{2}\left[K_{a}\left(\frac{C_{o l}}{m}\right)\right]^{0.5}\right) .
$$

in which $R_{S}, C_{e, 0}$ and $C_{o l}$ represent the mole concentrations of chain scission, initial ester bonds and short chains, $k_{1}$ and $k_{2}$, the kinetic rate constants for non-catalytic 
and autocatalytic hydrolysis reactions, $\alpha$ and $\beta$ are constants indicating the nature of chain scissions (random or end scissions), $K_{a}$ represents the equilibrium constant for acid disassociation of the carboxylic ends, and $m$, the average degree of polymerization of the short chains which is generally taken as 4 [18]. The production rate of short chains due to polymer chain cleavage is given by [18, 19]

$$
\frac{d R_{o l}}{d t}=\alpha \beta\left(\frac{R_{s}}{C_{e, 0}}\right)^{\beta-1} \frac{d R_{s}}{d t} .
$$

in which $R_{o l}$ is the concentration of ester bonds of all the short chains. The average molecular weight $M_{n}$ of the polymer is calculated using [18, 19]

$$
M_{n}=M_{n, 0} \frac{1-\alpha\left(\frac{R_{s}}{C_{e, 0}}\right)^{\beta}}{1+N_{d p, 0}\left(\frac{R_{s}}{C_{e, 0}}-\frac{\alpha}{m}\left(\frac{R_{s}}{C_{e, 0}}\right)^{\beta}\right)}
$$

in which $M_{n, 0}$ is the initial molecular weight and $N_{d p, 0}$, the degree of polymerisation of the polymer.

\subsection{Introduction of erosion in the degradation model}

Interior erosion and surface erosion are considered here. Following Gopferich [14] two rules for erosion of a pixel at the interior of an implant are used: (i) molecular weight at the pixel must be below a critical value, and (ii) the pixel must be in contact with an eroded neighbour. This can be written as

$$
\begin{aligned}
& S(i, j, t)=0 \text { if } \\
& M_{n}(i, j, t)<M_{n, \text { critical }} \text { and the pixel has an eroded neighbour. }
\end{aligned}
$$

Here, $M_{n, \text { critical }}$ represents the critical molecular weight. This erosion model leads to a sudden release of oligomers when the critical molecular weight is reached. While some polymers do show this sudden mass loss in the degradation experiment, other polymers show a gentler trend in mass loss $[12,21]$. The slow mass erosion can be captured by introducing an incubation period, $t_{\text {inc }}$, in the model. A pixel of low 
molecular weight only changes its state from $S=1$ to $S=0$ after being in contact with an eroded neighbour for an incubation period of $t_{\text {inc }}$.

For some polymers, surface erosion starts as soon as the polymer is in contact with the surrounding medium. The volumetric erosion rate is given by [22]

$$
\frac{d V(t)}{d t}=-B
$$

in which $\frac{d V(t)}{d t}$ represents the volume of material lost per unit surface area per unit time, and $B$ is a material constant. For a pixel in contact with the surrounding medium, $S(i, j, t)$ is changed to zero when its volume is reduced to zero through Eq. 7 .

\subsection{Rate equation for oligomer and drug diffusion}

Oligomers and solubilised drug molecules are capable to diffuse through the polymeric matrix. Because the typical size of drug particles is much smaller than the characteristic diffusion distance in a drug-loaded polymer, the actual morphology of the drug particles are ignored and the drug-loaded polymer is treated as a continuum solid. Assuming Fick's law for diffusion, the oligomer and drug concentrations, $C_{o l}$, and $C_{d r u g}$ are governed by [23]

$$
\frac{\partial C_{K}}{\partial t}=\frac{\partial R_{K}}{\partial t}+\frac{\partial}{\partial x}\left(D_{K, x} \frac{\partial C_{K}}{\partial x}\right)+\frac{\partial}{\partial y}\left(D_{K, y} \frac{\partial C_{K}}{\partial y}\right)
$$

in which the subscript $K$ refers to either ol, to represent oligomer, or $d r u g$, to represent drug. $R_{K}$ is the rate of short chain production or drug dissolution and $D_{K, x}$ and $D_{K, y}$ are the diffusion coefficients in $\mathrm{x}$ and $\mathrm{y}$-directions. The current oligomer concentration $C_{o l}$ is coupled back to Eq. 3 .

In the diffusion model, polymer morphology and pores are treated in an effective manner using effective diffusion coefficients. These diffusion coefficients depend on the local porosity of the polymer matrix. They are also controlled by the way in which the pores are connected locally, i.e. the diffusion coefficients have very different values in different directions. To consider the orientation dependence, two "porosities", $\varepsilon_{x}$ and $\varepsilon_{y}$, are introduced such that [18] 


$$
D_{K, x}=D_{K, \text { poly }}+\left(1.3 \varepsilon_{x}^{2}-0.3 \varepsilon_{x}^{3}\right)\left(D_{K, \text { pore }}-D_{K, \text { poly }}\right)
$$

and

$$
D_{K, y}=D_{K, \text { poly }}+\left(1.3 \varepsilon_{y}^{2}-0.3 \varepsilon_{y}^{3}\right)\left(D_{K, \text { pore }}-D_{K, \text { poly }}\right) .
$$

in which $D_{K, \text { poly }}$ and $D_{K, \text { pore }}$ represents the diffusion coefficients in the non-degraded bulk polymer and water filled pores respectively. A local "porosity" in a particular direction is defined as the fraction of eroded pixels over a pre-defined number of pixels, $2 n_{\text {range }}$, vertical to that direction. $\varepsilon_{x}$ and $\varepsilon_{y}$ are therefore calculated as

$$
\begin{aligned}
& \varepsilon_{x}(i, j, t)=\frac{1}{2 \times n_{\text {range }}+1} \sum_{j=j-n_{\text {range }}}^{j=j+n_{\text {range }}} S(i, j, t) \\
& \varepsilon_{y}(i, j, t)=\frac{1}{2 \times n_{\text {range }}+1} \sum_{i=i-n_{\text {range }}}^{i=i+n_{\text {range }}} S(i, j, t)
\end{aligned}
$$

The mass loss $W_{t}$ is calculated by summation of mass losses due to short chain diffusion, $W_{d}$, and erosion, $W_{e}$, such as

$$
W_{t}=W_{d}+W_{e}
$$

Similarly, drug release, $M_{t}$ is calculated as summation of drug release due to drug diffusion, $M_{d}$, and release carried by polymer erosion, $M_{e}$, such as

$$
M_{t}=M_{d}+M_{e}
$$

\subsection{The numerical procedure and different behaviours of mass loss}

The central finite difference scheme is used for spatial discretization for the second term on the right hand side in Eq. 8. The time integration is performed by using the direct Euler scheme, i.e. values of $R_{S}$ and $C_{K}$ at $\mathrm{t}=\mathrm{t}+\Delta t$ are calculated as

$$
R_{S}(t+\Delta t)=R_{S}+\frac{d R_{s}}{d t} \Delta t
$$




$$
C_{K}(t+\Delta t)=C_{K}+\frac{d C_{K}}{d t} \Delta t
$$

It is assumed that $C_{o l}(i, j)=0$ and $R_{S}(i, j)=0$ at $t=0$. Perfect sink conditions are assumed at the interface between the polymer and its surrounding medium, which requires that $C_{o l}=0$ at the interface. Numerical convergence is obtained by gradually increasing the number of pixels and decreasing the time step.

Considering an infinitively large plate, Fig. 2 shows the calculated profiles of mass loss due to (a) diffusion of short chains out of the plate, (b) surface erosion, (c) interior erosion and (d) interior erosion with an incubation period. The reduction to these individual mechanisms of the model is achieved by only switching on the individual mechanism concerned. The following model parameters are used to generate Fig. 2 which are broadly based on experimental data from [24]. The values of molecular weights are set as $M_{n, \text { mean }}=7.5 \times 10^{4} \mathrm{~g} / \mathrm{mol}, M_{n, s t \_d e v}=2.0 \times 10^{4} \mathrm{~g} / \mathrm{mol}, M_{n, \text { critical }}=2.7 \times 10^{4}$ $\mathrm{g} / \mathrm{mol}$ and $M_{\text {unit }}=65 \mathrm{~g} / \mathrm{mol}$. The size of the representative unit of the plate is taken as $80 \times 320 \mu \mathrm{m}$. $D_{\text {ol,poly }}=5.0 \times 10^{-15} \mathrm{~m}^{2} /$ week and $D_{\text {ol,pore }}=1.0 \times 10^{-5} \mathrm{~m}^{2} /$ week. The rate of surface erosion is set as $B=6.5 \times 10^{-14} \mathrm{~m}^{2} /$ week and hydrolysis reaction constants are set as $k_{1}=5.0 \times 10^{-6}$ week $^{-1}, k_{2}=2.0 \times 10^{-2} \mathrm{~m}^{3} \mathrm{~mol}^{-1}$ week $^{-1}$. The number of pixels in $\mathrm{x}$ - and $y$-directions, $n_{x}, n_{y}$, are set as $250 \times 1000$. The values of parameter $C_{e, 0}, m, \alpha$ and $\beta$ were obtained from Pan et al. [18].

For the sake of comparison, all the simulations were terminated at 30 days. Fig. 2(a) shows the mass loss from the reaction-diffusion equation. As can be seen, the short chain diffusion reaches only $0.3 \%$ of the total polymer weight. Fig. 2(b) shows the mass loss due to surface erosion. The surface erosion starts at $t=0$, with a constant rate which leads to continuous thinning of the plate. $15 \%$ mass loss is reached with the specified erosion rate. Fig. 2(c) shows the mass loss when the interior erosion is the dominant mechanism. It has an almost zero mass loss followed by a dramatic release of oligomers. Fig. 2(d) shows the mass loss profile due to interior erosion with an incubation period of 0.5 week. As can be seen, the mass loss of each induvial mechanism exhibits a distinctive behaviour. Diffusion of the short chains makes very little contribution to the mass loss because the typical diffusion coefficient of the short chains is very small $[16,19]$. Surface erosion leads to a linear mass loss over time. The small jumps in the figure are a characteristic of the discrete erosion model and 
can be explained as that a pixel cannot be eroded until its volume reduces to zero. On the other hand, interior erosion presents a burst phase in the release of oligomers. The zero mass loss at the early stages of interior erosion is because of the time taken for pixels to satisfy the two criteria of erosion. Once these criteria are satisfied, polymer erodes promptly leading to a dramatic mass loss.

The introduction of an incubation period for interior erosion produces the mass loss trend shown Fig. 2(d) that is most widely observed in the literature. The trend is achieved by assuming that a polymer of very low molecular weight cannot dissolve immediately when being in contact with water. Consequently, this study shows that the incubation behaviour is a general and important factor that has to be considered when understanding the degradation of aliphatic polyesters.

\section{Case studies of fitting the model with experimental data}

The model was applied to two experimental studies in the literature to demonstrate its capacity and provide an insight into the mechanisms underlying each case.

\subsection{Case Study A}

Grizzi et al. [8] carried out a set of experiments using PLA50 in order to compare the degradation behaviour of plates of different sizes. Large and thin plates were allowed to age under iso-osmolar phosphate buffer solution at $\mathrm{pH} 7.4$ and $37^{\circ} \mathrm{C}$. The dimensions of the samples used were $15 \times 10 \times 2 \mathrm{~mm}$ and $15 \times 10 \times 0.3 \mathrm{~mm}$. The initial molecular weight was $20,000 \mathrm{gmol}^{-1}$ and $34,000 \mathrm{gmol}^{-1}$ for the thick plates and thin films respectively. The molecular weight and mass loss were measured as degradation proceeds. Fig. 3(a) presents a schematic picture of a 3-dimensional plate. Since the plate is comparatively long in the z-direction, in our model matter transport is ignored in that direction and a 2-dimensional simplification were used. Due to symmetry only one quarter of the plate, shown using the shaded region in Fig. 3(a), was considered. As indicated in Fig. 3(b), the quarter of the plate is divided into uniform square pixels of $n_{x} \times n_{y}=200 \times 1000$ for the thick plate and $30 \times 1000$ for the thin plate. Our numerical studies showed that these densities of pixels ensure numerical convergence. Hydrolytic chain cleavage, oligomer diffusion and interior erosion are included in the model. Surface erosion was excluded. 
Table 1 provides the model parameters used in the fittings. These parameters are divided into two groups: Group I refers to the measured data and data that cannot be varied to fit a particular set of experimental data. The measured data includes $M_{n \text {,mean }}$ [8], $M_{n, s t \_d e v}$ [8], $M_{\text {unit }}$ [25], $C_{e, 0}$ [25], $K_{a}$ [18] and $D_{\text {ol,pore }}$ [26]. Parameter $m$ represents the average number of repeating units of oligomers which is taken as 4 . Parameters $\alpha$ and $\beta$ reflect the random nature of the polymer chain cleavage and are taken from Pan et al. [18]. $M_{n, \text { critical }}$ is determined from the experimental data of Grizzi et al. [8] such that they corresponds to the times at which significant mass loss occurred in the experiment. Group II refers to the data that were varied to fit the experimental data. $k_{1}$ and $k_{2}$ are the non-catalytic and autocatalytic rate constants for the hydrolysis reaction. $D_{o l, p o l y}$ is the diffusion coefficient of the oligomers in the non-degraded polymer. In a previous work [18], a comprehensive study on parameter sensitivity was performed for the polymer degradation model. In the newly added erosion model the parameters are unique for a particular set of experimental data. Hence, the model sensitivity to these parameters is clear.

Table 1 Model parameters to fit the experimental data obtained by Grizzi et al. [8]

\begin{tabular}{lllll}
\hline & $\begin{array}{l}\text { Model } \\
\text { parameters }\end{array}$ & Units & $\begin{array}{l}\text { Thick } \\
\text { plate }\end{array}$ & $\begin{array}{l}\text { Thin } \\
\text { plate }\end{array}$ \\
\hline \multirow{6}{*}{ Group I } & $M_{n, \text { mean }}$ & $\mathrm{g} \mathrm{mol}^{-1}$ & $2 \times 10^{4}$ & $3.4 \times 10^{4}$ \\
& $M_{n, \text { st_dev }}$ & $\mathrm{g} \mathrm{mol}^{-1}$ & $5 \times 10^{3}$ & $1.5 \times 10^{3}$ \\
& $M_{\text {unit }}$ & $\mathrm{g} \mathrm{mol}^{-1}$ & 72 & 72 \\
& $m$ & no unit & 4 & 4 \\
& $\alpha$ & no unit & 0.4 & 0.4 \\
& $\beta$ & no unit & 1 & 1 \\
& $C_{e, 0}$ & $\mathrm{~mol} \mathrm{~m}^{-3}$ & 17300 & 17300 \\
& $K_{a}$ & no unit $^{2}$ & $1.35 \times 10^{-4}$ & $1.35 \times 10^{-4}$ \\
& $B$ & $\mathrm{~m}^{2}$ week & 0 & 0 \\
& $M_{n, \text { critical }}$ & $\mathrm{g} \mathrm{mol}^{-1}$ & $4.1 \times 10^{3}$ & $7.0 \times 10^{3}$ \\
& $D_{\text {ol,pore }}$ & $\mathrm{m}^{2}$ week $^{-1}$ & $1.0 \times 10^{-5}$ & $1.0 \times 10^{-5}$ \\
\hline \multirow{6}{*}{ Group II } & $k_{1}$ & week $^{-1}$ & $7.0 \times 10^{-5}$ & $7.0 \times 10^{-5}$ \\
& $k_{2}$ & $\mathrm{~m}^{3} \mathrm{~mol}^{-1}$ week $^{-1}$ & $1.0 \times 10^{-2}$ & $1.0 \times 10^{-2}$ \\
& $D_{\text {ol,poly }}$ & $\mathrm{m}^{2}$ week $^{-1}$ & $5.0 \times 10^{-15}$ & $5.0 \times 10^{-15}$ \\
\hline
\end{tabular}

The discrete symbols in Fig. 4 and 


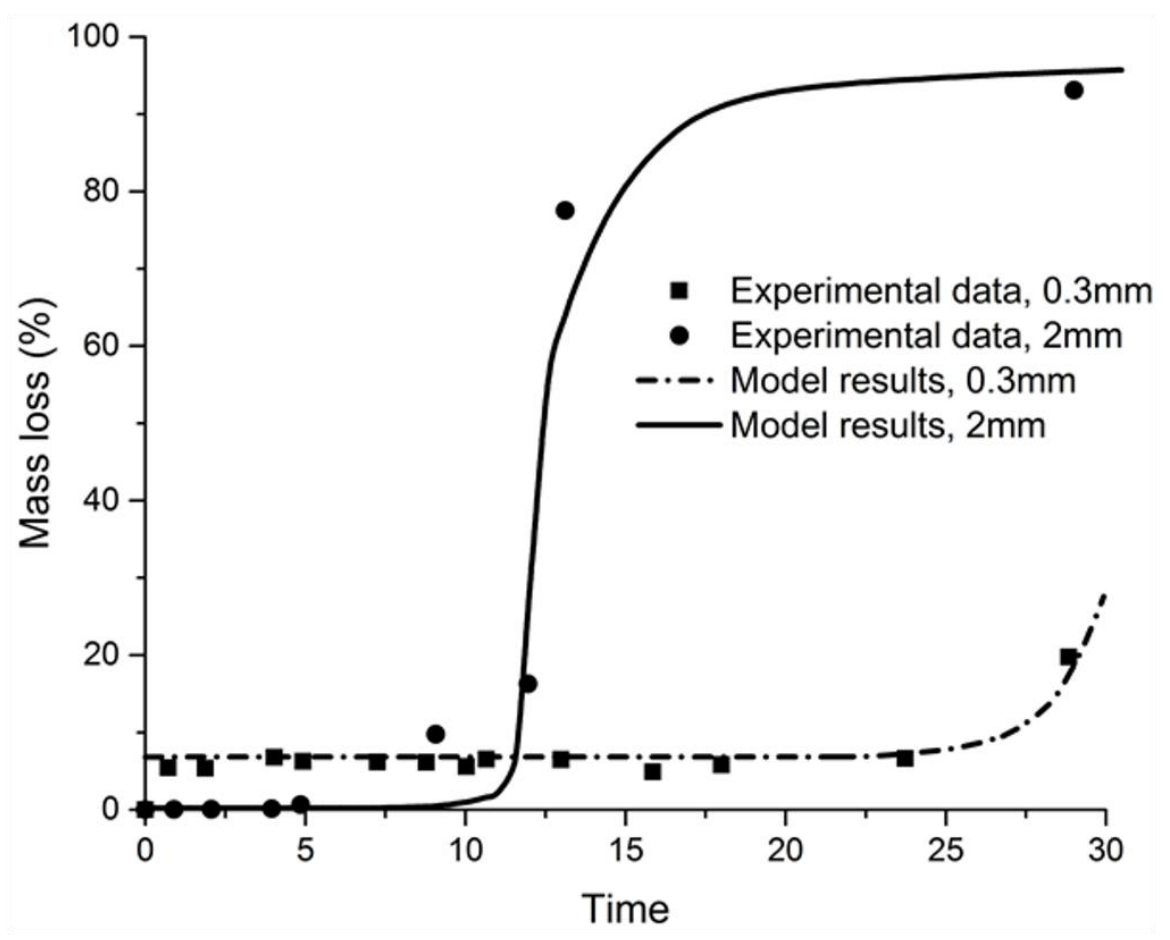

Fig. 5 reproduce the experimental data of the number averaged molecular weights and mass losses as functions of time, obtained by Grizzi et al. [8] . The fit of the model with the experimental data is shown using solid lines (for thick plate) and dashed dot lines (thin film) in these figures. It can be observed that the model fits the experimental data of the mass losses and average molecular weights reasonably well. The model clearly captures the size effect of the degradation. Considering Fig. 5, in the experiment the thin samples had a sudden mass loss of $7 \%$ at the beginning of the tests. This could only be due to dissolution of residual monomers and was treated as an initial condition of mass loss in the model. Very little mass loss was observed until week 24 after which a sudden mass loss occurred. The thick samples also showed very little mass loss until week 9 , which is then followed by a sudden mass loss reaching almost $100 \%$. This type of mass loss behaviour is very difficult to capture using a reaction-diffusion model. The polymer chain scissions simply cannot produce enough oligomers to give a mass loss of such quantity at the corresponding molecular weight. Only by considering interior erosion together with the hydrolytic chain scission, it is possible for the mathematical model to capture this mass loss behaviour. This means that interior erosion is the dominant mechanism controlling mass loss in these experiments. For the thin samples, there is only one experimental data point at week 28 showing significant mass loss. In the model a small incubation period of 0.2 week 
was used to give a gentle tread in mass loss toward 30 weeks. This is however arbitrary and not absolutely necessary because of the lack of experimental data.

Li et al. [27] conducted follow up degradation experiments on PLA/GA specimens and observed strong surface-centre differentiation during the degradation. A clear whitish layer of hard shell at the surface and a hollow interior were clearly observed at the end of their experiment. Li et al. [27] attributed this phenomenon to acid accumulation at the interior of the thick sample. The polymer degrades much faster at the core of the sample due to low $\mathrm{pH}$ generated by acid disassociation of the carboxylic end groups. This surface-centre differentiation has been clearly captured by our model, which is shown in Fig. 6. The pattern of erosion shown in Fig. 6 can be directly compared to those observed experimentally by Li et al. [27]. The white pixels represent non-eroded polymer and the black ones represent the eroded cells. No interior erosion occurs until week 11, because the inner pixels do not meet the two criteria of erosion (see Eq. 6). When the criteria are met, an interconnected network of eroded cells are quickly formed, which leads to a sudden release of material from interior of the sample. Burkersroda [28] related the break of the surface layer to a critical osmotic pressure inside the matrix due to the accumulation of erosion products. This study shows that no such osmotic pressure is necessary for the heterogonous breaking up of the samples.

\subsection{Case Study B}

The release kinetics of drugs dissolved in a polymeric matrix can generally follow a multiphasic release mechanism, including a diffusion-controlled phase, a degradation controlled phase and an erosion-controlled phase. Depending on the polymer type, some of these phases may be absent. In this case study, we demonstrate that model presented in this paper can be used to identify the underlying mechanisms of polymer degradation and drug release. Wang et al. [24] studied the release of sirolimus from bi-layer and tri-layer biodegradable films made of supporting layer(s) and a drug eluting layer. The supporting layer is made of PLLA and the drug-eluting layer is made of PLGA. $1 \%$ and $2 \%$ wt $\%$ drug loadings were considered in the study to analyse the effect of drug loadings on the release profile. The thicknesses of the drug loaded layer were about $80 \mu \mathrm{m}$. Degradation and drug release experiments were performed in the release medium (5\% Dichloromethane (DMSO) $+95 \% \mathrm{pH} 7.4 \mathrm{PBS}$ ) at $37^{\circ} \mathrm{C}$. The 
molecular weight, mass loss and drug release were measured as functions of time. Moreover, the surface morphology of drug-eluting layer was monitored during the degradation.

Fig. 7 provides a schematic picture of a cross-section for a stent with a drug-loaded layer. This is a simplistic representation of a stent structure. It is appropriate here because drug release is local to the drug-loaded layer and the overall structure of the stent is irrelevant to the drug release profile. The supporting layers are ignored because they are irrelevant to the drug release. Because the strut is long in the direction normal to the paper, matter transport in that direction is ignored and the problem is treated as 2-dimensional one. In fact Wang et al. [24] used two flat layers of one supporting layer and one drug-eluting layer in their experiment to simulate drug release from a stent. Fig. 7 (c) shows the representative unit of the drug-eluting layer that is modelled in this work. A set of square pixels of $n_{x} \times n_{y}=80 \times 2500$ were used for a representative unit of $80 \times 2500 \mu \mathrm{m}$.

Table 2 Parameters used in the model to fit with experimental data obtained by

Wang et al. [24]

\begin{tabular}{llll}
\hline & $\begin{array}{l}\text { Model } \\
\text { parameters }\end{array}$ & Units & Values \\
\hline & $M_{n, \text { mean }}$ & $\mathrm{g} \mathrm{mol}^{-1}$ & $7.5 \times 10^{4}$ \\
& $M_{n, \text { st_dev }}$ & $\mathrm{g} \mathrm{mol}^{-1}$ & $2.0 \times 10^{4}$ \\
& $M_{\text {unit }}$ & $\mathrm{g} \mathrm{mol}^{-1}$ & 65 \\
& $m$ & no unit & 4 \\
Group I & $\alpha$ & no unit & 0.4 \\
& $\beta$ & no unit & 1 \\
& $C_{e, 0}$ & mol m $\mathrm{m}^{-3}$ & 20615 \\
& $K_{a}$ & no unit $^{2}$ & $1.35 \times 10^{-4}$ \\
& $B$ & $\mathrm{~m}^{2}$ week & 0 \\
& $M_{n, \text { critical }}$ & $\mathrm{g} \mathrm{mol}^{-1}$ & $2.0 \times 10^{4}$ \\
& $D_{\text {ol,pore }}$ & $\mathrm{m}^{2}$ week & $1.0 \times 10^{-5}$ \\
& $D_{\text {drug,pore }}$ & $\mathrm{m}^{2}$ week $^{-1}$ & $1.0 \times 10^{-5}$ \\
\hline \multirow{6}{*}{ Group II } & $k_{1}$ & week $^{-1}$ & $5.0 \times 10^{-6}$ \\
& $k_{2}$ & $\mathrm{~m}^{3}$ mol $^{-1}$ week $^{-1}$ & $2.5 \times 10^{-2}$ \\
& $D_{\text {ol,poly }}$ & $\mathrm{m}^{2}$ week $^{-1}$ & $5.0 \times 10^{-15}$ \\
& $D_{\text {drug,poly }}$ & $\mathrm{m}^{2}$ week $^{-1}$ & $5.0 \times 10^{-17}$ \\
\hline
\end{tabular}


Table 2 presents model parameters used in the fitting. Again, the parameters are divided into two groups as Group I, which are obtained from measured data and Group II which were varied to fit the experimental data. The measured data including $M_{n \text {,mean }}$ [24], $M_{n, \text { st_dev }}$ [24], $M_{\text {unit }}$ [25], $C_{e, 0}$ [25] and $K_{a}$ [18] are taken from the related references. $D_{o l, p o r e}$ and $D_{\text {drug,pore }}$ are diffusion coefficients in liquid filled pores for oligomers and drug respectively which are simply taken as very large numbers [26]. The values of $k_{1}, k_{2}, D_{\text {ol,poly }}$ and $D_{\text {drug,poly }}$ were obtained by fitting the model calculations with the experimental data. The initial oligomer concentration is set as $C_{o l, 0}=0$.

Figs 8 and 9 show comparison between the model calculation and experimental data for molecular weight and mass loss as functions of time. In Fig. 9 the experimental data shows a fast initial mass loss of about $3.8 \%$ which is used as the initial condition in our model. After 15 days, an almost linear behaviour in mass loss was observed in the experiment. As discussed in section 2.4, this behaviour can only be modelled using an incubation period for polymer dissolution. An incubation period of 1.25 week gives the fittings shown in the figure. Consequently, this study shows that the incubation behaviour is a key factor that controls the degradation in this particular case. Fig. 10 illustrates the model fitting of drug release with the experimental data. Sirolimus release of two loadings of 1 and $2 \%$ were simulated respectively. The very small drug loadings justify the ignorance of phase separation between drug particles and the polymer, which is assumed in the drug diffusion model. The release profiles overlap for the two loadings and only the results for $1 \%$ loading are shown in Fig. 10. It can be observed from Figs 9 and 10 that drug release is very closely related to polymer mass loss. Because the incubation behaviour is critical to understand the mass loss, it is also critically important to the understanding of drug release. In this particular case it is polymer erosion rather than drug diffusion that control the drug release. In fact various drugs used in coronary stents tend to have large molecular weights. Their diffusion in the drug-loaded layer is therefore very slow and consequently their release cannot be modelled satisfactorily without considering polymer erosion.

Fig. 11 shows the computer simulated microstructure of eroded drug-eluting layer at 21 days. White pixels indicate the non-eroded polymer matrix whereas black pixels represent the eroded volume. Many horizontal tunnels are formed starting at the right 
surface of the drug-eluting layer and propagating into the interior. The simulated microstructure can be compared with those observed experimentally in [24] by Wang et al. In their experiment, isolated surface cavities were observed which correspond to the horizontal tunnels in Fig. 11.

\section{Conclusion}

A mathematical model is presented in this paper to simulate the hydrolytic degradation and erosion process of aliphatic polyesters. As far as we are aware, this is the first time that the mechanisms of autocatalytic hydrolytic degradation, oligomer diffusion, and surface and interior erosion are all considered in a single mathematical model. Our study highlights several important issues when modelling the degradation and drug release. In general, it is important to link hydrolytic degradation and erosion processes in order to understand different kinds of mass loss and drug release trends. In particular it is revealed that (a) it is unlikely to model mass loss larger than $10 \%$ using a reaction-diffusion model because polymer chain scissions cannot provide enough oligomers to be released from an implant; (b) experimental data often shows that a critical molecular weight exists below which a sudden mass (and drug release) occurs. It is critically important to embed this information into a mathematical model; (c) an incubation behaviour for polymer dissolution is an important factor when understanding mass loss and drug release for some polymers; and (d) the release of drugs used in coronary stent can only be modelled satisfactorily by taking into account of polymer erosion because the diffusion of these drugs is very slow.

\section{Acknowledgement}

K.S. gratefully acknowledges the financial support provided by Turkey Ministry of National Education, YLSY program, for the research on this article. The authors would also like to state that this research used the ALICE High Performance Computing Facility at the University of Leicester.

\section{References}

[1] Ikada Y, Tsuji H. Biodegradable polyesters for medical and ecological applications. Macromolecular rapid communications 2000;21:117-32.

[2] Domb AJ, Kumar N. Biodegradable polymers in clinical use and clinical development: John Wiley \& Sons; 2011.

[3] Bastioli C. Handbook of biodegradable polymers: iSmithers Rapra Publishing; 2005.

[4] Rezaie HR, Bakhtiari L, Öchsner A. Biomaterials and Their Applications: Springer; 2015. 
[5] Frank A, Kumar Rath S, Boey F, Venkatraman S. Study of the initial stages of drug release from a degradable matrix of poly(d,I-lactide-co-glycolide). Biomaterials 2004;25:813-21.

[6] Gopferich A, Langer R. Modeling of polymer erosion. Macromolecules 1993;26:4105-12.

[7] Siepmann J, Göpferich A. Mathematical modeling of bioerodible, polymeric drug delivery systems. Advanced drug delivery reviews 2001;48:229-47.

[8] Grizzi I, Garreau H, Li S, Vert M. Hydrolytic degradation of devices based on poly(DL-lactic acid) size-dependence. Biomaterials 1995;16:305-11.

[9] Zygourakis K. Development and temporal evolution of erosion fronts in bioerodible controlled release devices. Chemical Engineering Science 1990;45:2359-66.

[10] Siepmann J, Faisant N, Benoit JP. A new mathematical model quantifying drug release from bioerodible microparticles using Monte Carlo simulations. Pharmaceutical Research 2002;19:1885-93. [11] Chen YH, Zhou SW, Li Q. Mathematical modeling of degradation for bulk-erosive polymers: Applications in tissue engineering scaffolds and drug delivery systems. Acta Biomaterialia 2011;7:1140-9.

[12] Li SM, Garreau H, Vert M. Structure-property relationships in the case of the degradation of massive aliphatic poly-( $\alpha$-hydroxy acids) in aqueous media. Journal of Materials Science: Materials in Medicine 1990;1:123-30.

[13] Zhang T, Zhou S, Gao X, Yang Z, Sun L, Zhang D. A multi-scale method for modeling degradation of bioresorbable polyesters. Acta biomaterialia 2017;50:462-75.

[14] Gopferich A. Polymer bulk erosion. Macromolecules 1997;30:2598-604.

[15] Husmann M, Schenderlein S, Lück $M$, Lindner $H$, Kleinebudde P. Polymer erosion in PLGA microparticles produced by phase separation method. International Journal of Pharmaceutics 2002;242:277-80.

[16] Gleadall A, Pan J, Kruft M-A, Kellomäki M. Degradation mechanisms of bioresorbable polyesters. Part 1. Effects of random scission, end scission and autocatalysis. Acta biomaterialia 2014;10:2223-32. [17] Gleadall A, Pan J, Kruft M-A, Kellomäki M. Degradation mechanisms of bioresorbable polyesters. Part 2. Effects of initial molecular weight and residual monomer. Acta Biomaterialia 2014;10:2233-40. [18] Pan J. Modelling degradation of bioresorbable polymeric medical devices: Elsevier; 2014.

[19] Wang Y, Pan J, Han X, Sinka C, Ding L. A phenomenological model for the degradation of biodegradable polymers. Biomaterials 2008;29:3393-401.

[20] Han X, Pan J. A model for simultaneous crystallisation and biodegradation of biodegradable polymers. Biomaterials 2009;30:423-30.

[21] Li S, Garreau H, Vert M. Structure-property relationships in the case of the degradation of massive poly ( $\alpha$-hydroxy acids) in aqueous media. Journal of Materials Science: Materials in Medicine 1990;1:198-206.

[22] Thombre AG, Himmelstein KJ. Modelling of drug release kinetics from a laminated device having an erodible drug reservoir. Biomaterials 1984;5:250-4.

[23] Sevim K, Pan J. A Mechanistic Model for Acidic Drug Release Using Microspheres Made of PLGA 50: 50. Molecular Pharmaceutics 2016;13:2729-35.

[24] Wang X, Venkatraman SS, Boey FY, Loo JS, Tan LP. Controlled release of sirolimus from a multilayered PLGA stent matrix. Biomaterials 2006;27:5588-95.

[25] Han X, Pan J. Finite element analysis of degradation of biodegradable medical devices. OA Biotechnology 2 (3) 2013;22.

[26] Stewart PS. Diffusion in Biofilms. Journal of Bacteriology 2003;185:1485-91.

[27] Li SM, Garreau H, Vert M. Structure-property relationships in the case of the degradation of massive poly ( $\alpha$-hydroxy acids) in aqueous media. Journal of Materials Science: Materials in Medicine 1990;1:131-9.

[28] von Burkersroda F, Schedl L, Gopferich A. Why degradable polymers undergo surface erosion or bulk erosion. Biomaterials 2002;23:4221-31. 


\section{Figures}

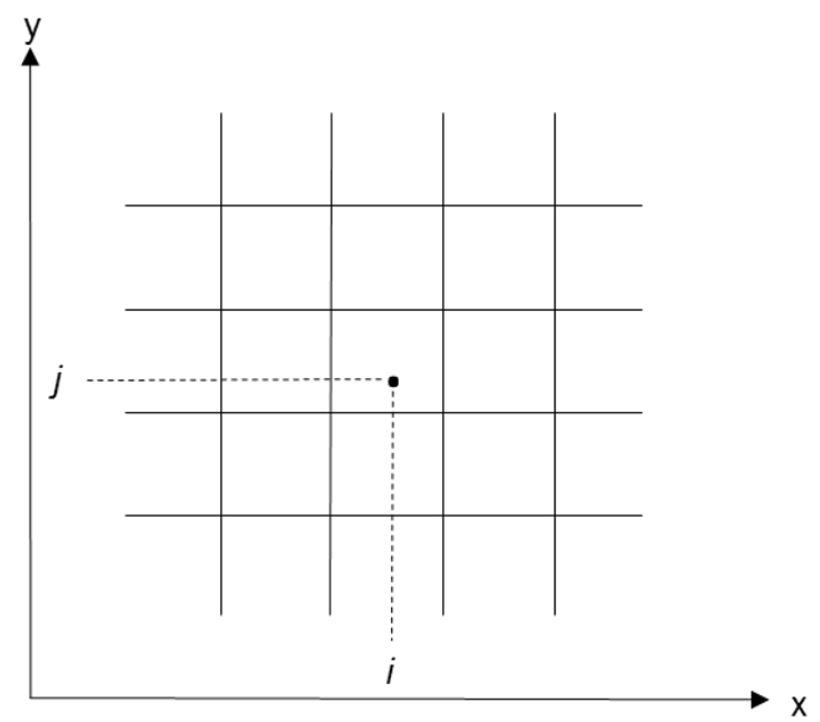

Fig. 1. Schematic illustration of discretised polymer.
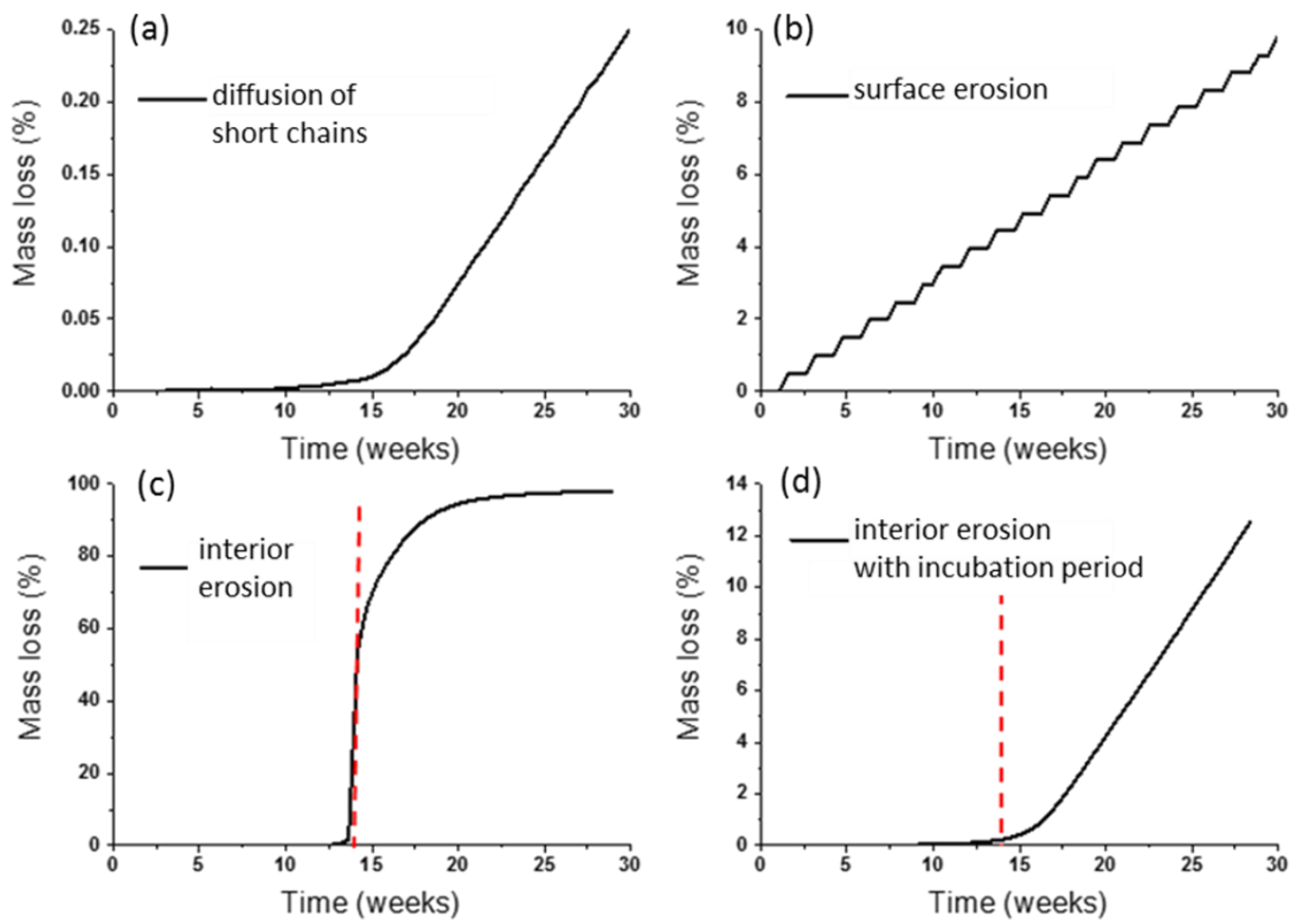

Fig. 2. Mass loss profiles due to individual mechanisms. 


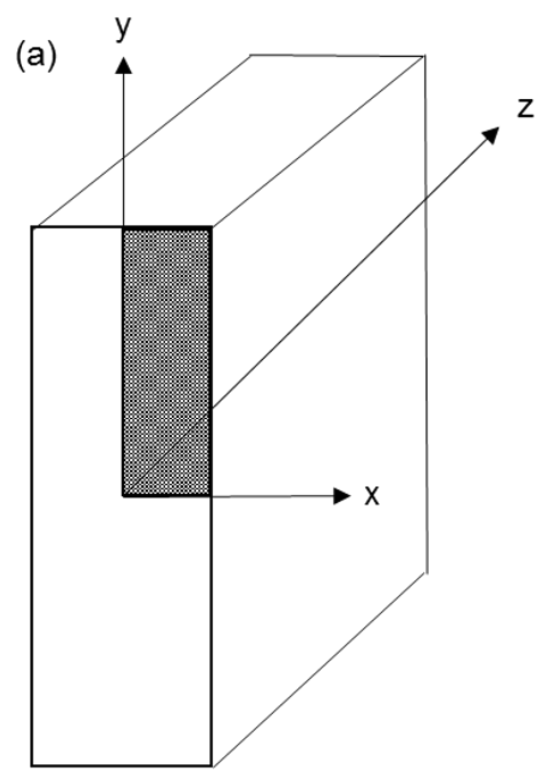

(b)

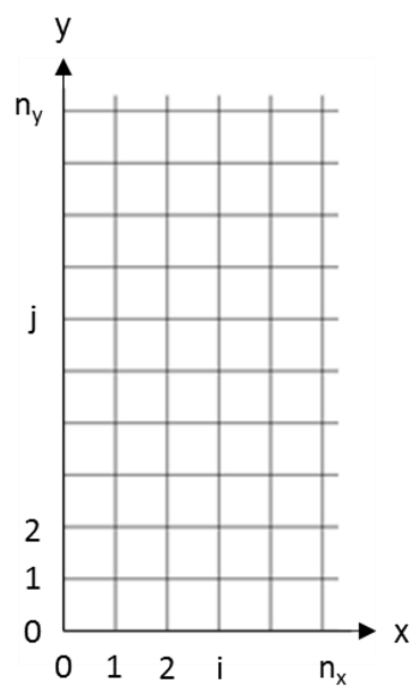

Fig. 3. Schematic of (a) 3-dimensional geometry; (b) 2-dimensional pixel grid used for numerical analysis.

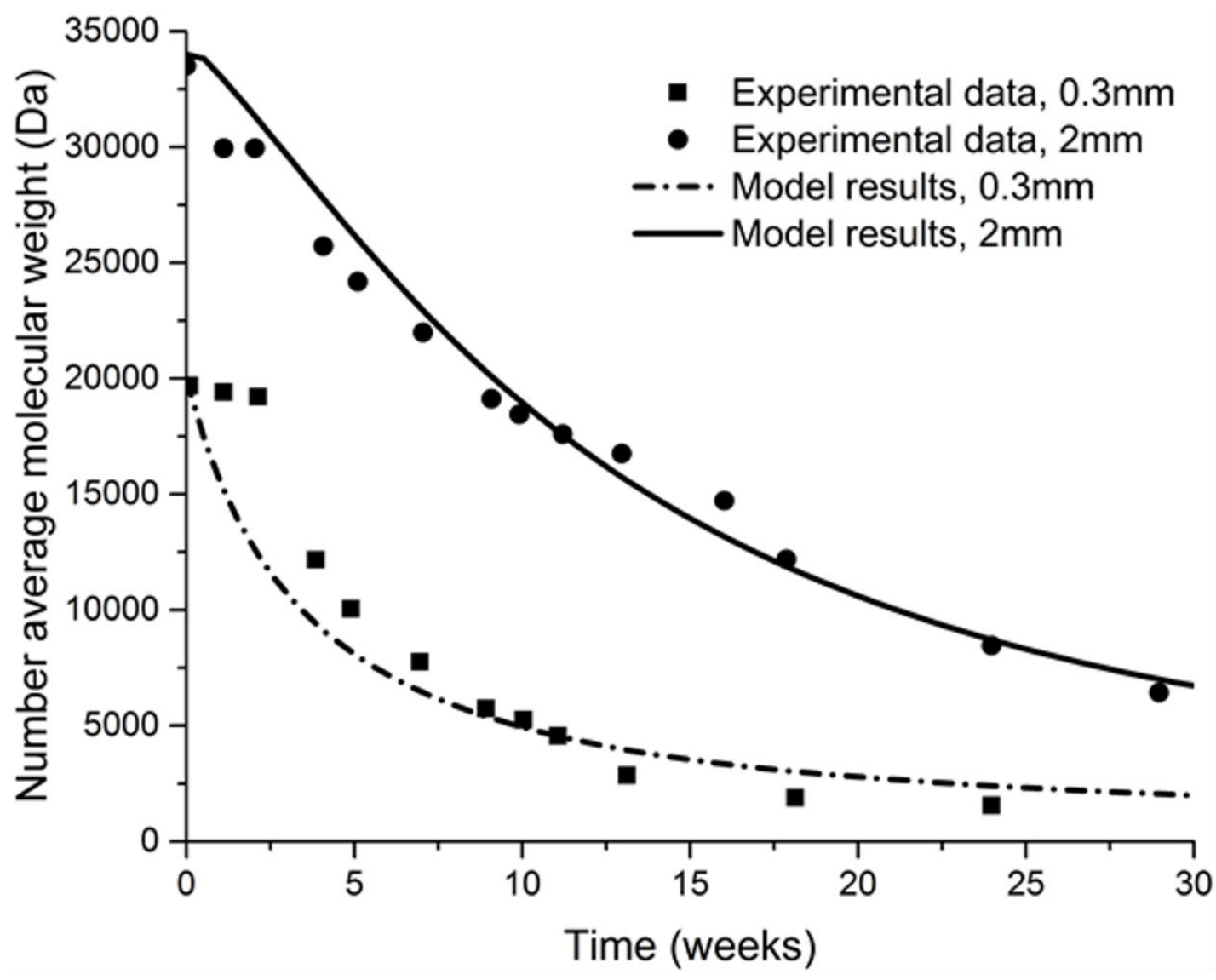

Fig. 4. Comparison of molecular weight change between model prediction and experimental data for different sizes. The solid lines represent the model predictions with the discrete symbols representing the experimental data [8]. 


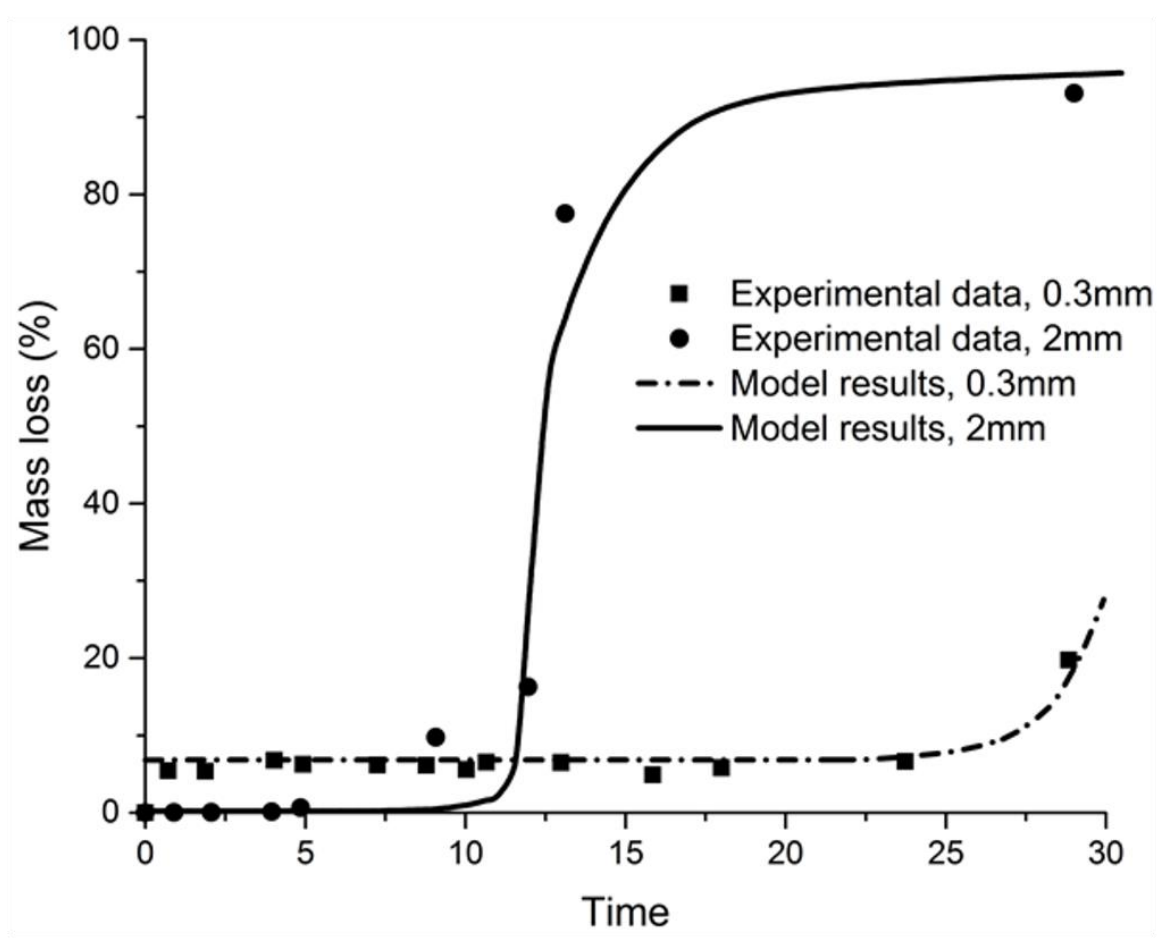

Fig. 5. Comparison of molecular mass loss between model prediction and experimental data for different sizes. The solid lines represent the model predictions with the discrete symbols representing the experimental data [8].
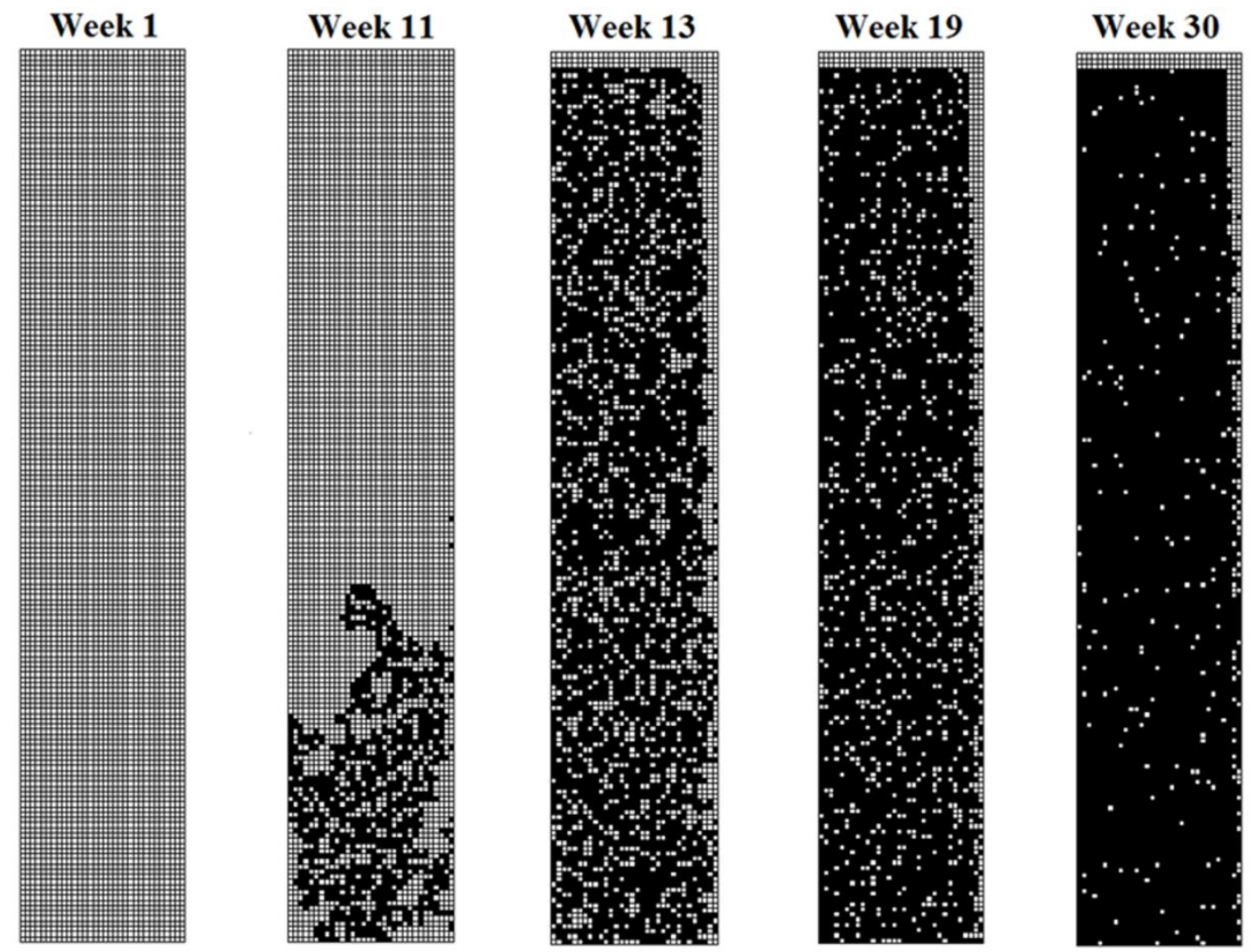
Fig. 6. Temporal evaluation of simulated the polymer matrix which can be directly compared to those obtained experimentally by Li et al. [27].

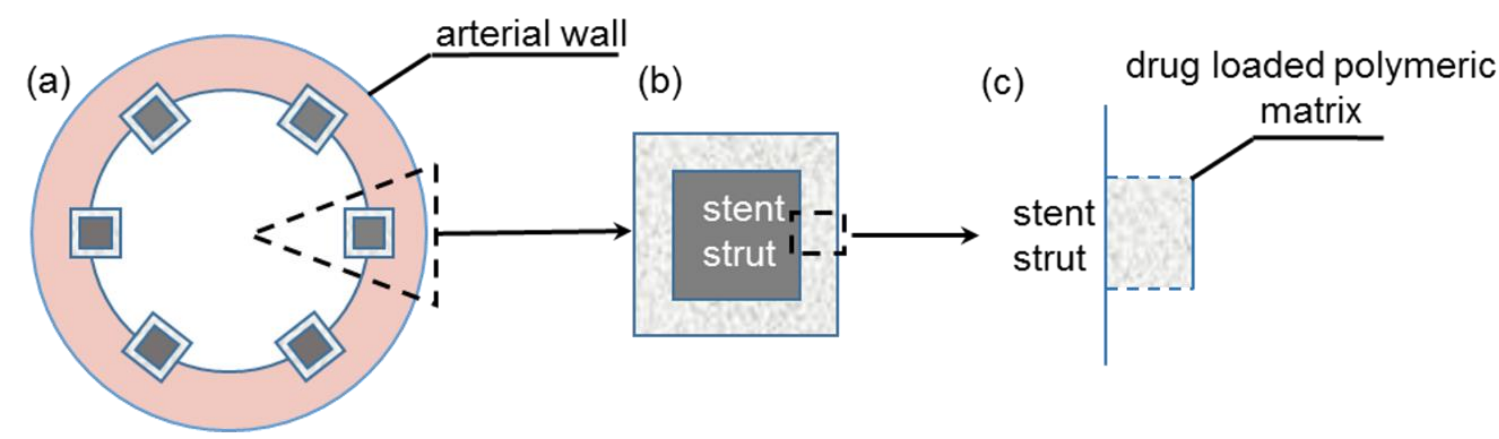

Fig. 7 (a) Cross section of an implant stent in coronary artery, (b) schematic of a single stent strut with drug-loaded polymer matrix and (c) schematic of the drug loaded polymer matrix which is modelled in the current paper.

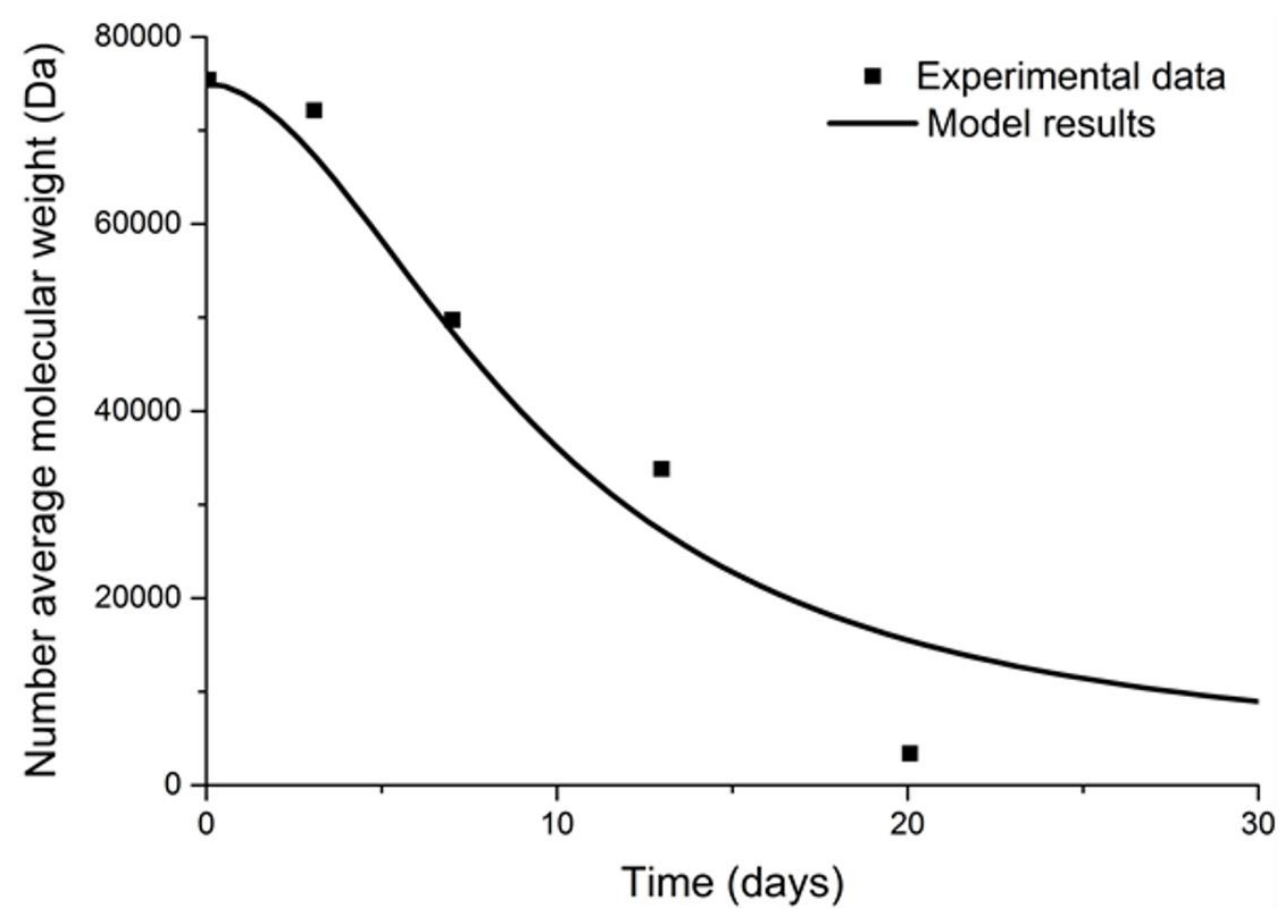

Fig. 8. Comparison of molecular weight decrease of PLGA between model prediction and experimental data. The solid line represents the model predictions with the discrete symbols representing the experimental data [24]. 


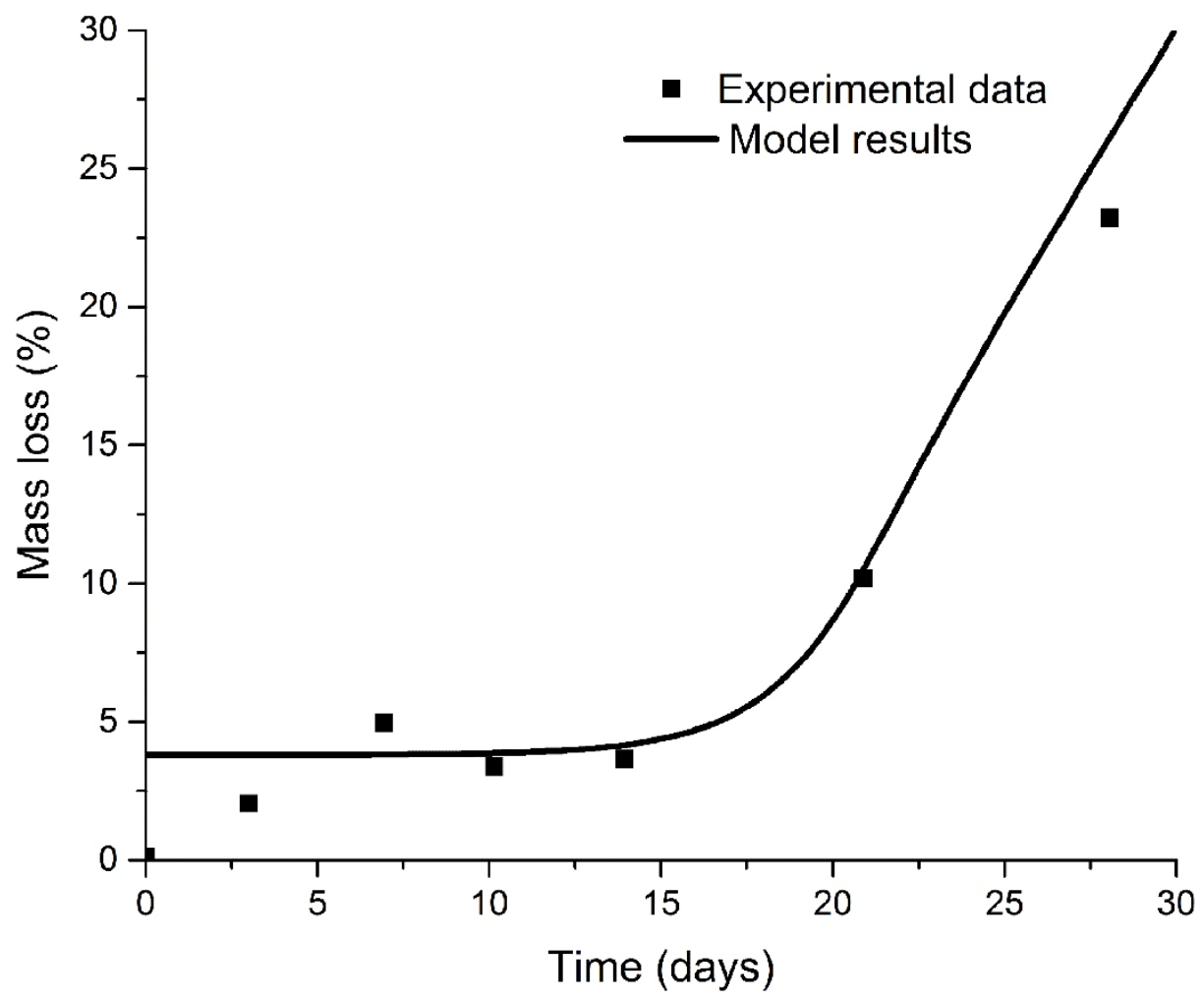

Fig. 9. Comparison of mass loss of PLGA between model prediction and experimental data. The solid line represents the model predictions with the discrete symbols representing the experimental data [24].

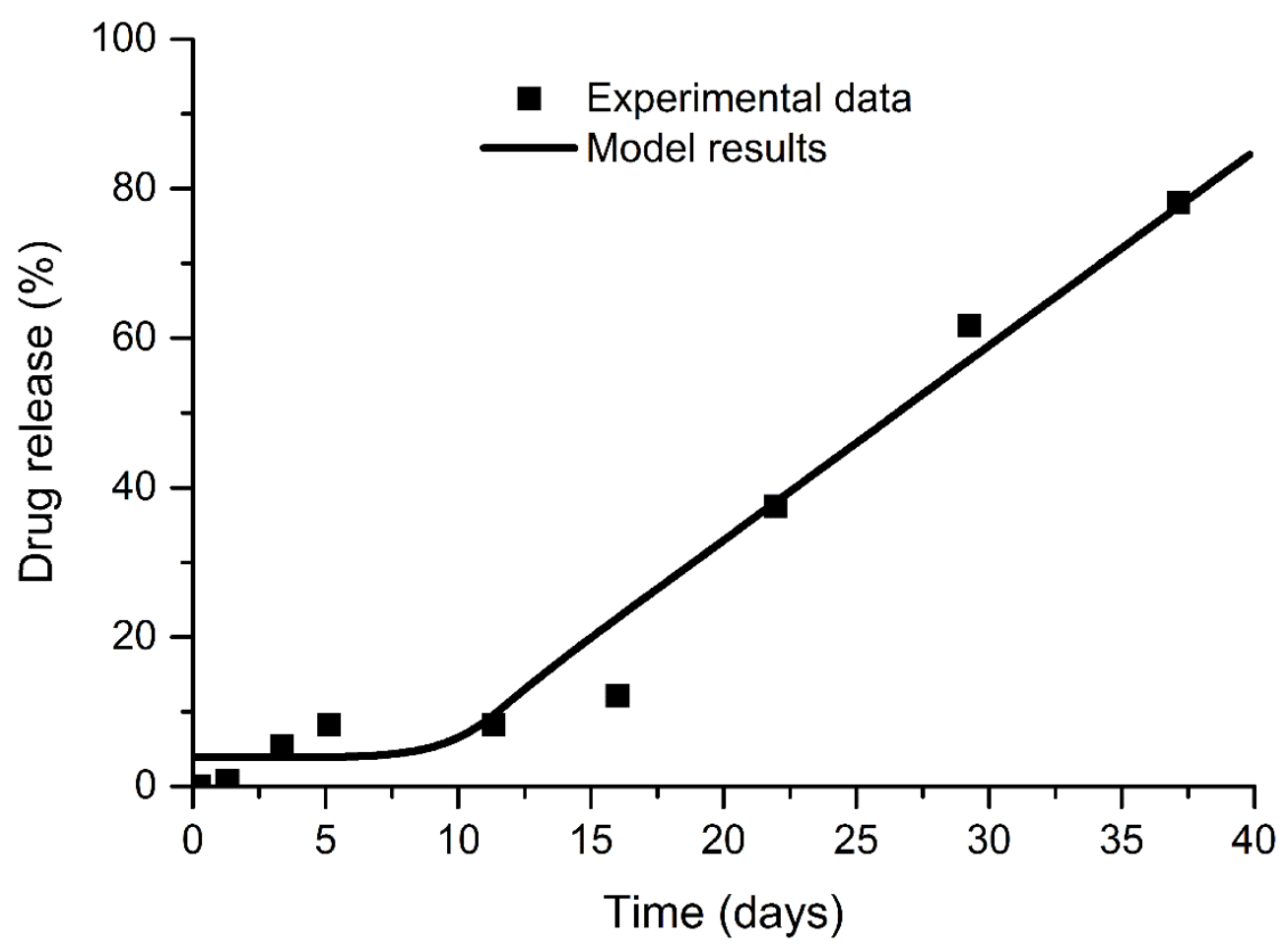

Fig. 10. Comparison of sirolimus release from PLGA between model prediction and experimental data. The solid line represents the model predictions with the discrete symbols representing the experimental data [24]. 
Published on Acta Biomaterialia, 8 Nov 2017, https://doi.org/10.1016/j.actbio.2017.11.023

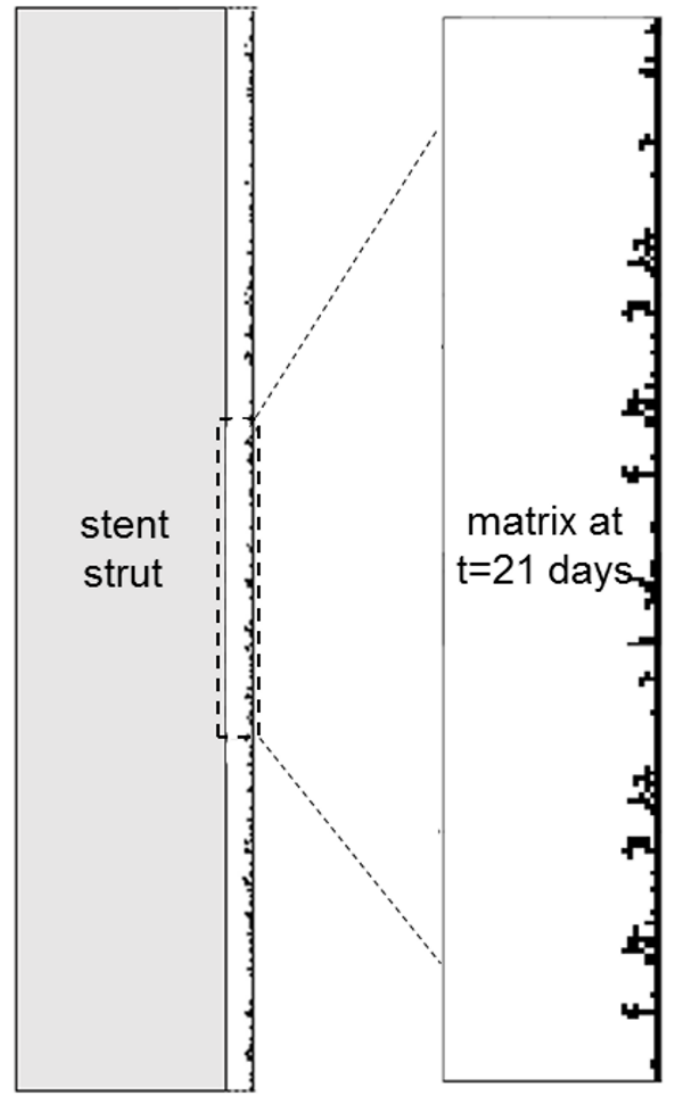

Fig.11. Simulation results for drug loaded polymer matrix at $t=21$ days. 